\title{
Structure and Properties of Hexadecyltrimethylammonium Chloride Monolayers in Contact with Oil Films with Different Thicknesses
}

\author{
Daniel J. V. A. dos Santos* and Jose A. N. F. Gomes \\ REQUIMTE/Departamento de Quimica da Faculdade de Ciencias da Universidade do Porto, \\ R. Campo Alegre 687, 4169-007 Porto, Portugal \\ Received: February 11, 2004; In Final Form: May 14, 2004
}

\begin{abstract}
We report a study on the effects of the amount of oil (100, 300, and 1372 1,2-dichloroethane molecules) spread on a hexadecyltrimethylammonium (CTA) chloride monolayer adsorbed on water. There are some related experimental results, namely, by neutron reflection on the Langmuir interface (air/water interface), that could be used carefully to understand the behavior of monolayers adsorbed at liquid/liquid interfaces. With this work we make a bridge between the Langmuir and the liquid/liquid interfaces. The addition of a small amount of DCE molecules changes dramatically various properties, namely, the distribution of the width of the chains, the distribution of the distance between the chain heads and tails, and the distribution of the tilt angle. On the other hand, the in-plane mean square distribution and the diffusion coefficient of the nitrogen atom are very similar in the systems studied, except for the system containing the largest amount of DCE molecules, which corresponds to the water/DCE interface.
\end{abstract}

\section{Introduction}

The study of the adsorbed monolayers and bilayers is a very vast subject due to the richness of systems that can be produced. ${ }^{1-3}$ Although the Langmuir interface (air/water interface) is one of the most studied, it is far from being the one with the greatest technological or industrial applications. On the other hand, the study of liquid/liquid interfaces is relatively recent mainly because the classical experimental techniques cannot give useful information on interfaces buried inside condensed phases. ${ }^{4}$ With the advent of new spectroscopic techniques (second-harmonic and sum frequency generation) $)^{5,6}$ or the use of neutron reflection techniques, ${ }^{7}$ the system behavior can now be interpreted on the basis of the information at the molecular level. Recently, Walker and co-workers ${ }^{8-11}$ used a very promising technique that uses second-harmonic generation to study the response of molecular rulers (solvent-sensitive cromophores) to the environmental changes occurring at liquid/ liquid and solid/liquid interfaces.

The Monte Carlo and molecular dynamics techniques also play an important role, since they can be used to connect the micro and thermodynamic point of view and because there is still some information that is obtainable by simulation, but not by the state-of-the-art experimental techniques. Using these techniques, several authors were successful at studing liquid/ liquid interfacial phenomena. ${ }^{12-21}$

Liquid/liquid interfaces are usually studied by simulation techniques, by sum frequency spectroscopy, ${ }^{6,22-24}$ and by some electrochemical techniques, namely, cyclic voltametry. The neutron reflection technique, although very important, is unable to give information on buried interfaces. Nevertheless, Thomas and co-workers have studied monolayers of dodecyltrimethylammonium and hexadecyltrimethylammonium (CTA) bromides, ${ }^{25}$ tetradecytrimethylammonium bromides, ${ }^{26}$ and pentaethylene glycol monolayers, ${ }^{27}$ all of them with an added layer

* Corresponding author. E-mail: dasantos@fc.up.pt. of dodecane. Although these studies are important per se, from the liquid/liquid interfaces study point of view, we would like to know if these systems can be regarded as containing a liquid/ liquid interface, whose properties are more or less dependent on the quantity of added oil or on the thickness of the film in contact with the monolayer, and what amount of oil should be added to attain the thermodynamic limit of a liquid/liquid interface. From another point of view, we would like to know what experimental information gathered by this technique can be directly used to better understand the behavior of monolayers adsorbed at liquid/liquid interfaces. On the other hand, the monolayer system with the added oil can also be regarded as a contaminated monolayer ${ }^{7,28}$ and used to study the contaminant effect upon the monolayer organization.

\section{Simulation Details}

We have adopted the same strategy, simulation parameters, techniques, and model potentials already used in a previous study of a hexadecyltrimethylammonium (CTA) chloride monolayer adsorbed at the Langmuir and at the water/DCE interfaces. ${ }^{21}$ We have used the SPC ${ }^{29}$ water potential and a modified model due to Böker and co-workers for the CTA, with the van der Waals and ligand potentials extracted from the CHARMM22 force field, except for the tails' dihedral angles that were substituted by the Ryckaert-Bellemans potential. The potential for the DCE was developed by Jorgensen and co-workers, ${ }^{30}$ and for the chlorine ion we have used the parameters obtained by Smith and Dang. ${ }^{31}$

Using a previously equilibrated system containing a CTA monolayer adsorbed at the water/DCE interface with 100 CTA, 100 chlorine ions, 4500 water molecules, and 1372 DCE molecules, ${ }^{21}$ we have removed the DCE molecules that were further away from the interface to obtain two systems, one containing only 100 DCE molecules and another containing 300 DCE molecules, all of them at the same coverage density of 45 $\AA^{2}$ per surfactant. We have used the same coverage density, since Thomas and co-workers ${ }^{7}$ verified that the incorporation 


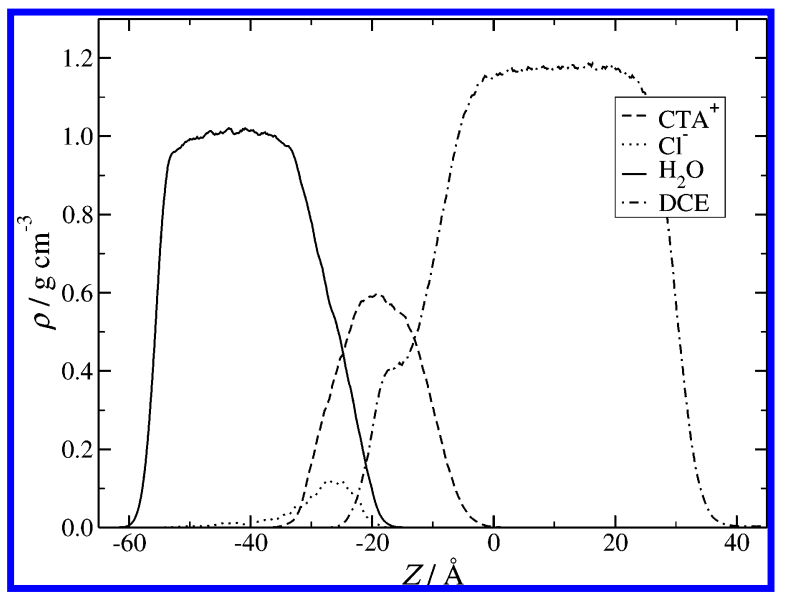

Figure 1. Average density profiles for the water, chloride ions, CTA ions, and DCE as a function of the box coordinate for the system containing 1372 DCE molecules.

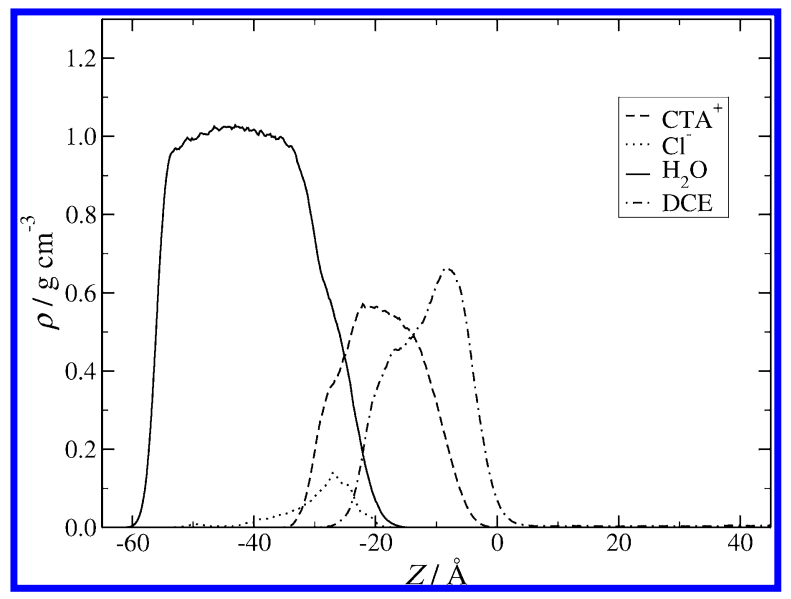

Figure 2. Average density profiles for the water, chloride ions, CTA ions, and DCE as a function of the box coordinate for the system containing 300 DCE molecules.

of alkane molecules in the CTA monolayers in equilibrium with the alkane vapor has a relativelly small effect on the surfactant density coverage.

These two systems were equilibrated for at least $750 \mathrm{ps, \text {and }}$ a production run just followed by 1000 ps. The particles' trajectories were written to a compressed disk file at every 32 steps and later analyzed.

\section{Results}

The three different systems containing 1372, 300, and 100 DCE molecules are represented by the density profiles in Figures 1,2 , and 3, respectively. For the system containing 1372 DCE molecules we can find in equilibrium with the monolayer a DCE slab with some bulk DCE. This system corresponds to a CTA monolayer adsorbed at the water/DCE interface. For the system containing 300 DCE molecules (a 3:1 DCE/CTA ratio) we can see that an important amount of DCE exists inside the monolayer and that the remainder covers the CTA tails. For the 100 DCE molecule system (a 1:1 DCE/CTA ratio) we can see that all the DCE molecules are incorporated inside the monolayer tails. In these last two systems, the amount of DCE is insufficient to achieve bulk type oil and can be understood as a very thin film of different thicknesses in equilibrium with a monolayer adsorbed on water. For all systems we have bulk water in equilibrium with the monolayer, but bulk DCE exists only in the system containing 1372 DCE molecules.

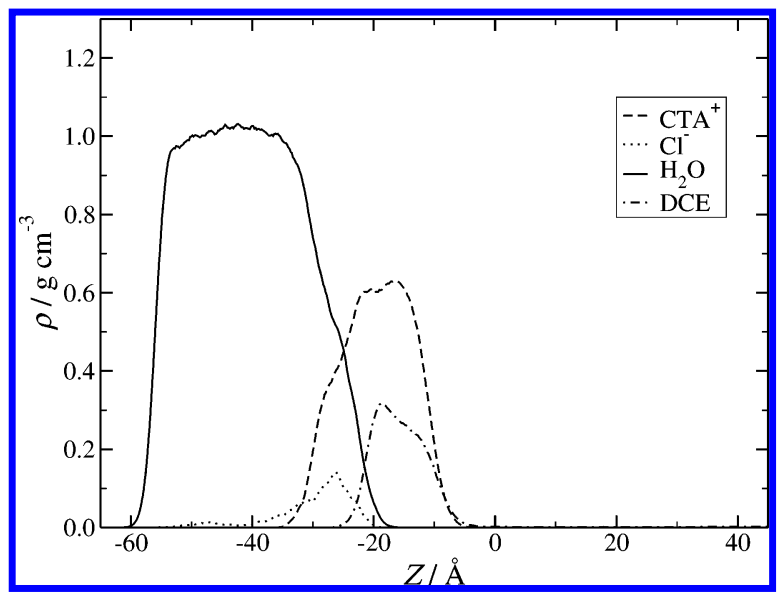

Figure 3. Average density profiles for the water, chloride ions, CTA ions, and DCE as a function of the box coordinate for the system containing 100 DCE molecules.

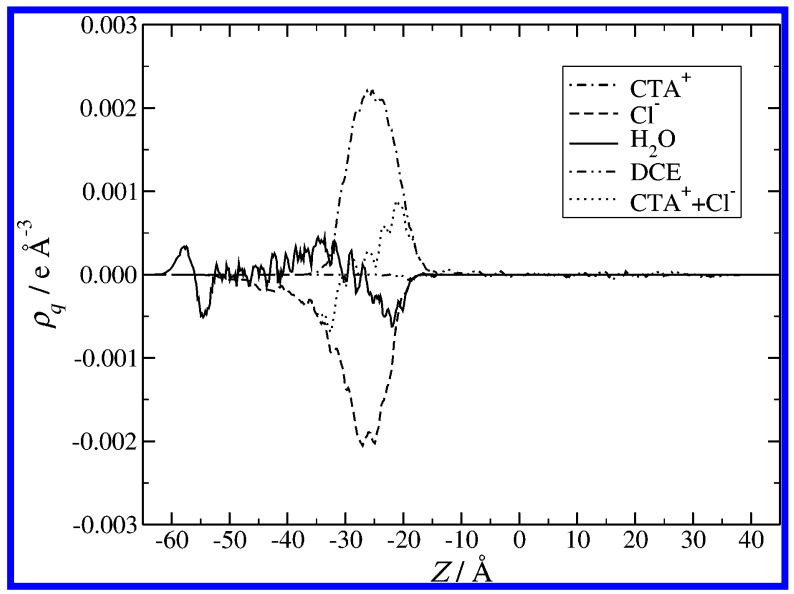

Figure 4. Average electron density profile for the water, chloride ions, CTA ions, DCE, and CTA plus chloride ions as a function of the box coordinate for the system containing 1372 DCE molecules.

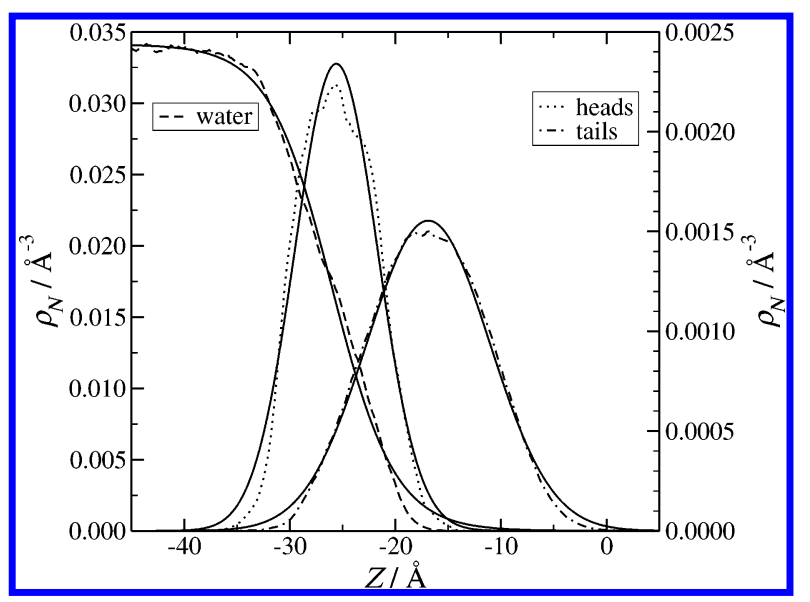

Figure 5. Profiles and fits for the water and CTA (heads and tails) number densities for the system containing 1372 DCE molecules. The water scale is located on the left-hand side, and the heads and tails scale is located on the right-hand side.

From Figure 4, we can look at the system containing 1372 DCE molecules from the average charge density profile of the species present. This property was also calculated for the other two systems and was found to behave similarly as in other systems with adsorbed CTA monolayers at different liquid/liquid interfaces. $^{21}$ It seems also to be rather insensitive to the differences in the amount of DCE molecules present in the systems. 
TABLE 1: Widths $\zeta$ and $\sigma$ of the Water and CTA Head and Chain Profiles ${ }^{a}$

\begin{tabular}{|c|c|c|c|c|c|c|}
\hline DCE & $\sigma_{\mathrm{h}} / \AA$ & $\sigma_{\mathrm{c}} / \AA$ & $\zeta / \AA$ & $\delta_{\mathrm{ch}} / \AA$ & $\delta_{\mathrm{hs}} / \AA$ & $\delta_{\mathrm{cs}} / \AA$ \\
\hline 1372 & $11.0 \pm 0.1$ & $16.45 \pm 0.07$ & $5.97 \pm 0.08$ & $8.72 \pm 0.06$ & $0.40 \pm 0.09$ & $9.12 \pm 0.08$ \\
\hline 300 & $10.8 \pm 0.2$ & $17.2 \pm 0.1$ & $5.96 \pm 0.08$ & $9.23 \pm 0.09$ & $0.4 \pm 0.1$ & $9.59 \pm 0.08$ \\
\hline 100 & $10.8 \pm 0.2$ & $14.7 \pm 0.1$ & $6.1 \pm 0.1$ & $8.8 \pm 0.1$ & $0.2 \pm 0.1$ & $9.1 \pm 0.1$ \\
\hline 0 & $11.0 \pm 0.2$ & $12.3 \pm 0.1$ & $6.53 \pm 0.09$ & $8.0 \pm 0.1$ & $0.2 \pm 0.1$ & $8.22 \pm 0.09$ \\
\hline
\end{tabular}

${ }^{a} \delta_{i j}$ are the separations between the distribution centers (c, chains; h, heads; s, water). The 0 DCE corresponds to the Langmuir interface, and the 1372 DCE corresponds to a water/DCE interface..$^{21}$

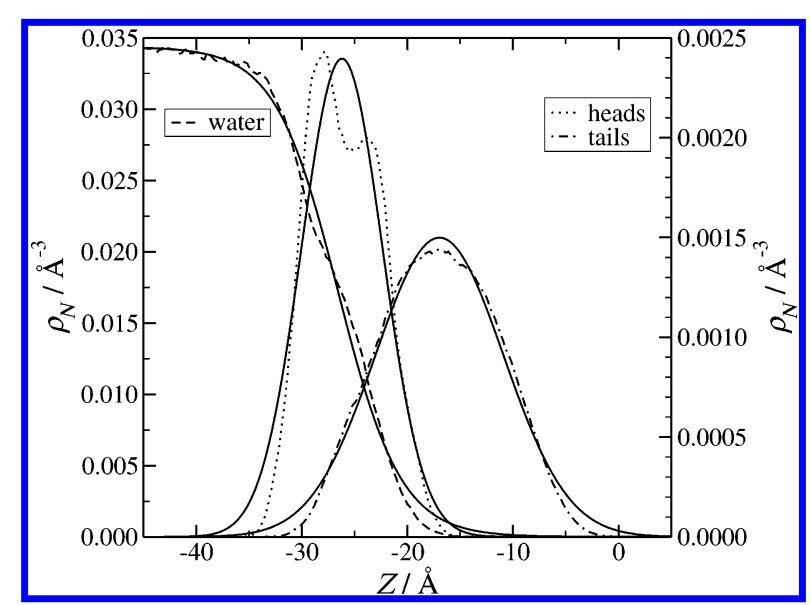

Figure 6. Profiles and fits for the water and CTA (heads and tails) number densities for the system containing 300 DCE molecules. The water scale is located on the left-hand side, and the heads and tails scale is located on the right-hand side.

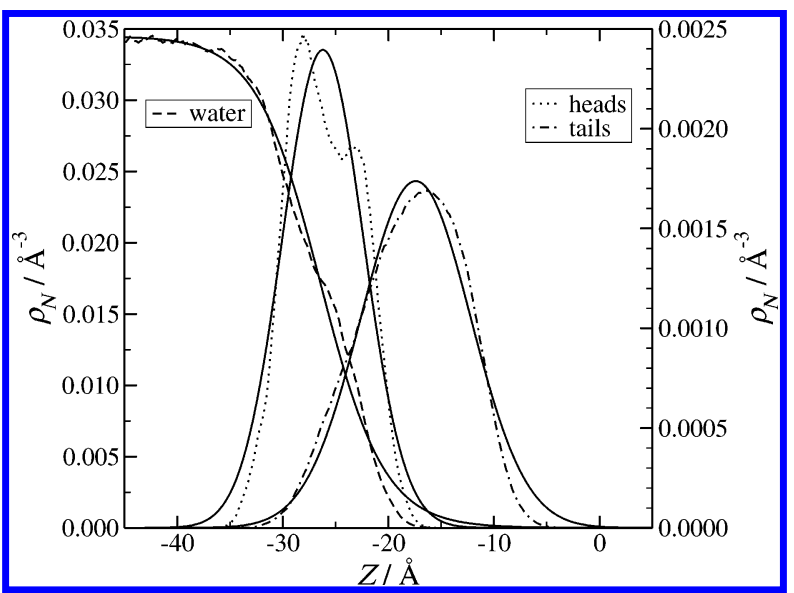

Figure 7. Profiles and fits for the water and CTA (heads and tails) number densities for the system containing 100 DCE molecules. The water scale is located on the left-hand side, and the heads and tails scale is located on the right-hand side.

The number density profiles for the water and CTA heads and tails are represented in Figures 5, 6, and 7 for the systems containing 1372, 300, and 100 DCE molecules, respectively. The fittings in these figures are for a hyperbolic tangent (water profile) and for a normal distribution (CTA heads and tails profiles). By directly inspecting these figures, we can see that unlike in the other systems, the distribution of the CTA tails of the system containing 100 DCE molecules is skewed. The parameters resulting from the fittings are presented in Table 1 (the results for the Langmuir interface obtained from a previous calculation $^{21}$ are also shown).

From Table 1, we can see that the width of the distribution of CTA heads is the same for all the systems. In opposition, the distribution width of the CTA tails is different among these systems. On average, we can say that the distribution width of the CTA tails increases as more DCE molecules are added to the system. Nevertheless, there is an unexpected behavior for

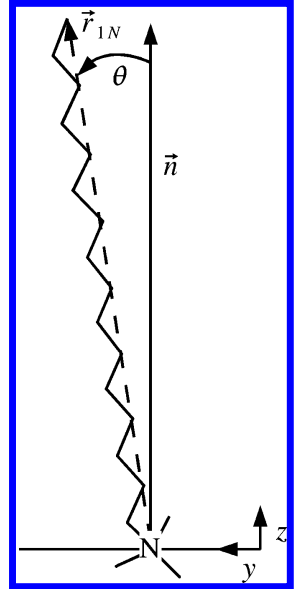

Figure 8. Definition of the tilt angle $\theta \cdot \vec{r}_{1 \mathrm{~N}}$ is the vector between the nitrogen atom and the terminal methyl group in the same CTA, and $\vec{n}$ is the surface normal vector. The surface plane is laid in the $y$ axis and the surface normal in the $z$ axis.

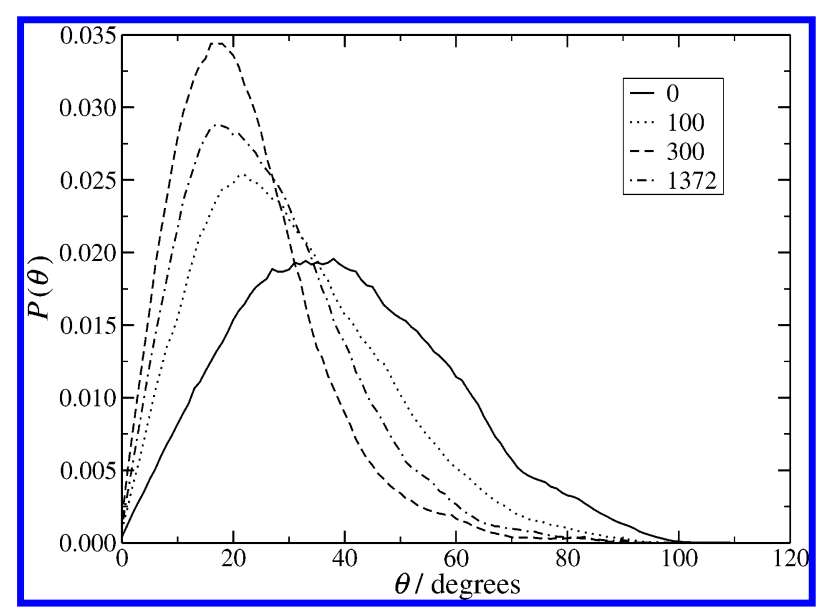

Figure 9. Tilt angle probability distribution of the CTA ions for the systems containing 1372, 300, 100, and 0 DCE molecules.

the system containing 300 DCE molecules, as one would expect an intermediate $\sigma_{\mathrm{c}}$ value (width of the chains distribution) when compared with the values obtained for the other two systems. For this system the $\sigma_{\mathrm{c}}$ value is the largest and can suggest a nonideal behavior for systems containing an oil film in equilibrium with a monolayer. For this property, only a small amount of DCE is necessary to produce a large increase in its value. Our results agree with those of Thomas and co-workers ${ }^{7}$ because they have also reported an increase in the thickness of the surfactant layer when dodecane is added.

The distance between the chains and heads distribution centers, $\delta_{\mathrm{ch}}$, and the distance between the chains and solvent distribution centers, $\delta_{\mathrm{cs}}$, are larger when there are DCE molecules in the system, and we can also say that only a small amount of DCE is necessary for the values to increase dramatically. It is interesting to note that for these properties the system containing only $100 \mathrm{DCE}$ and the system containing 1372 DCE molecules have similar values. The largest $\delta_{\mathrm{ch}}$ value 

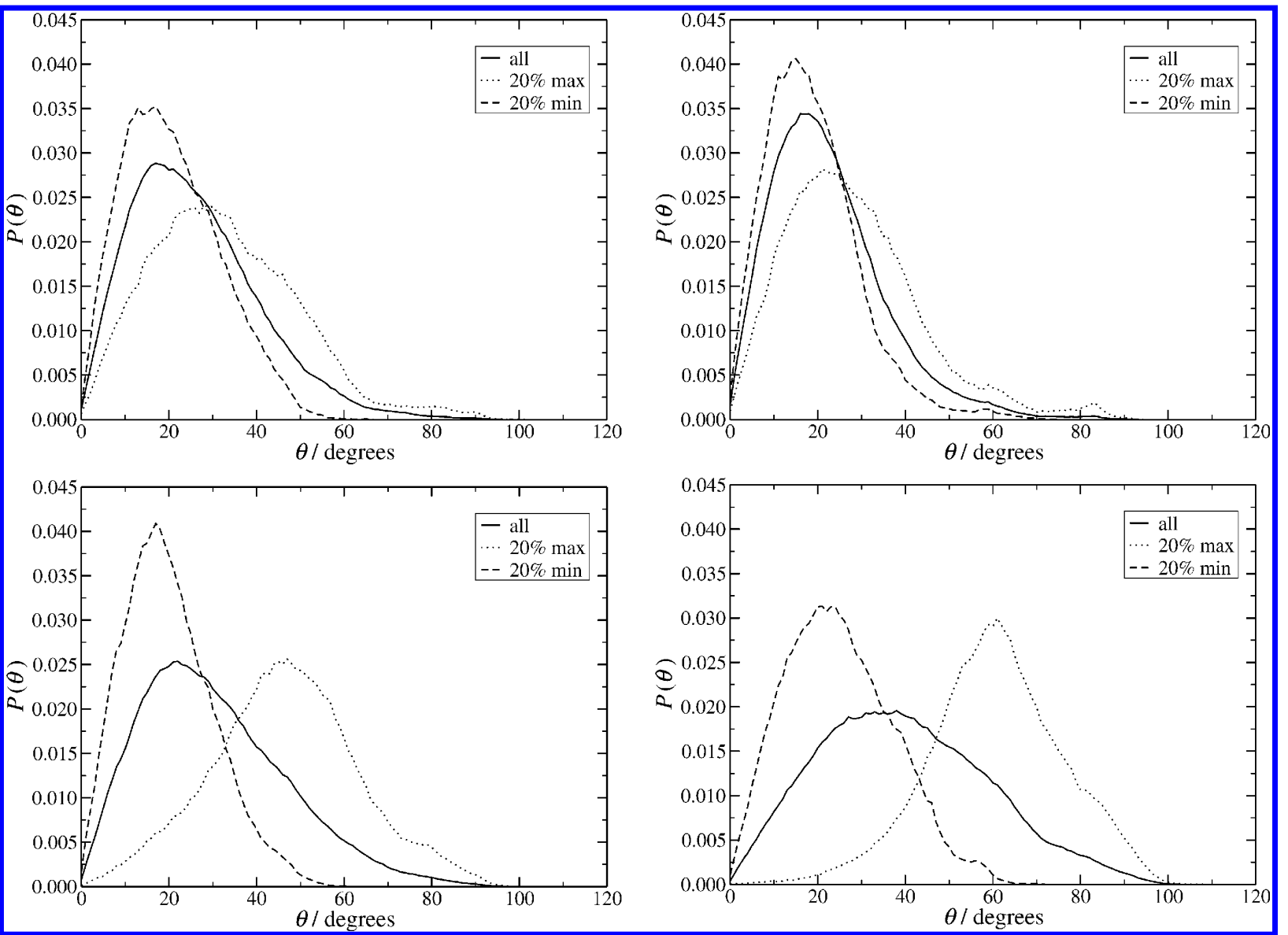

Figure 10. Tilt angle probability distribution of the CTA ions for the systems containing 1372 (top left), 300 (top right), 100 (bottom left), and 0 DCE molecules (bottom right). The $20 \% \mathrm{max} / \mathrm{min}$ refers to the $20 \mathrm{CTA}$ ions that have the nitrogen atom with a larger/smaller $z$ position (less/ more penetration in the water).

belongs to the system containing 300 molecules, and this suggests that the average tilt angle (angle between the normal vector of the interface and the vector between the nitrogen atom and the terminal methyl group in the same molecule) ${ }^{21}$ and, maybe also, the number of cis conformations along the CTA chains are smaller than for the other systems. In fact, as we will later show, both factors have a positive contribution for the existence of a smaller $\sigma_{\mathrm{c}}$ and $\delta_{\mathrm{ch}}$.

For the water number density distribution width, we have a larger value for the system without DCE molecules (Langmuir interface), and it is quite similar among the systems that contain DCE. This means that near the interface and for the Langmuir interface, the decrease in the number of water molecules with the box position is smaller; that is, the water interface is less sharp. On the other hand, the center of the distribution of the distance between the water and the CTA heads is equal at the Langmuir interface and for the system containing 100 DCE molecules, but it is larger for the other two systems. For these reasons, we can say that it is at the Langmuir interface where we can find the greatest contact between the water and the CTA heads.

In Figure 8 the tilt angle of a CTA molecule is defined, and in Figure 9, the tilt angle probability distributions ${ }^{21}$ for the Langmuir interface, for the water/DCE interface, and for the systems containing 100 and 300 DCE molecules are presented. We can see that 100 DCE molecules are enough to dramatically change this distribution, since the average tilt angle and the maximum probability angle are very similar to the values found for the system containing 1372 DCE molecules. For the systems containing DCE, the maximum probability angle is near $20^{\circ}$ and the average value is $31^{\circ}, 23^{\circ}$, and $27^{\circ}$ for the systems containing 100, 300, and 1372 DCE molecules, respectively. Although the maximum probability angle is almost constant among the systems containing DCE, the average value is smaller and the distribution is sharper for the system containing 300 DCE molecules. For the Langmuir interface, the maximum probability tilt angle is located near $40^{\circ}$, with the same average value.

By analyzing Figure 10, we can have a clear picture of the differences between the tilt angle distributions, namely, the differences between the tilt angle distributions of the CTA heads that penetrate more or less into the water slab. We can see that although a small quantity of DCE is sufficient to have an average tilt angle distribution similar to the one found for the system corresponding to the water/DCE interface, the same does not happen with the distributions of the CTA that penetrate more or less into the water layer. It is clearly seen that a 1:1 CTA to DCE ratio is insufficient to have similar distributions. This result shows that an amount of oil to completely cover the CTA tails is necessary to obtain small differences between the distribution of the CTA that penetrate more and less into the water layer.

In Figure 11, we present an analysis of the average percentage of trans conformations for every dihedral angle along the CTA chain. The smaller percentage of trans conformations is generally found for the system containing 100 DCE molecules, and the greatest percentage is found for the system containing $300 \mathrm{DCE}$ molecules, while the system containing 1372 DCE molecules has intermediate values. For this property, the system containing 300 DCE molecules presents also an unexpected behavior because it does not assume an intermediate position. Neverthe- 


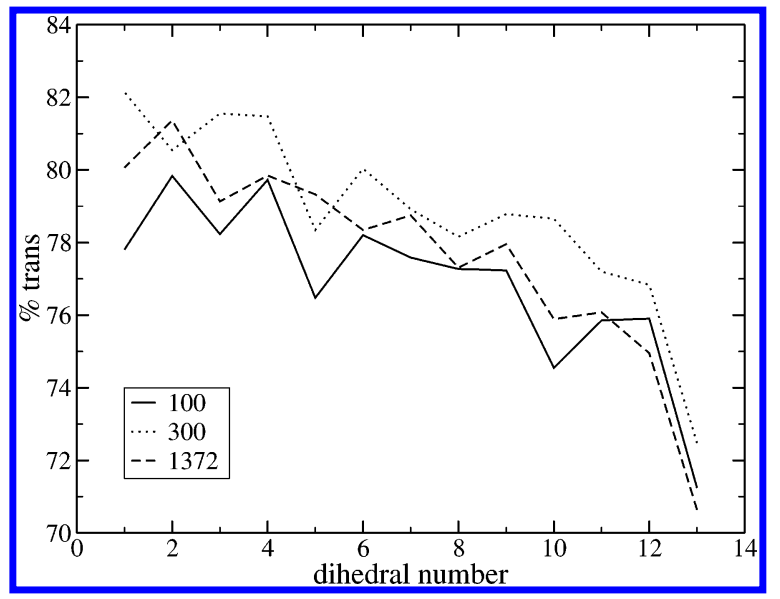

Figure 11. Percentage of trans conformations in the CTA hydrocarbon chains as a function of the dihedral number. On the left-hand side is the water slab and on the right the DCE slab.

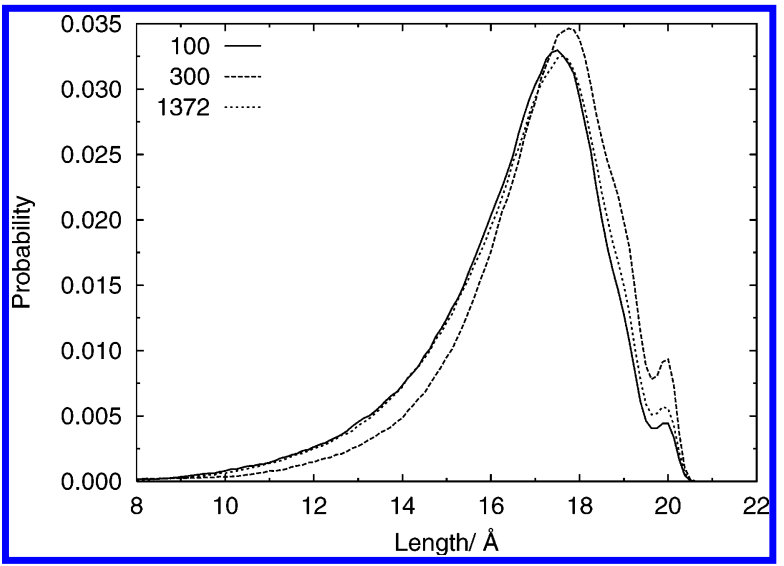

Figure 12. Average head to tail distance distribution.

less, for all systems the usual zigzag profile and the decrease in the percentage of trans conformations are observed when one goes along the CTA chain from the head until the terminal methyl group. A large percentage of trans conformations is linked to a large head to tail distance. As can be seen in Figure 12 , it is also for the system containing 300 DCE molecules that there is a larger probability of finding a more stretched tail. For this property, the systems containing 100 and 1372 DCE molecules have a similar probability distribution.

Several order parameters can be calculated that give local information about a molecular axis deviation from the monolayer normal. The chain order parameter is defined as ${ }^{21,32}$

$$
S_{z z}=\frac{1}{2}\left\langle 3 \cos \theta_{i} \cos \theta_{j}-1\right\rangle
$$

where $\theta_{i}$ is the angle between the $i$ th molecular axis and the monolayer normal ( $z$ axis) and is related to the local order of the long molecular axis. The bond order parameter is defined using the bond vector as the molecular axis. The experimentally observed deuterium order parameter can be obtained using the following equation:

$$
S_{\mathrm{CD}}=\frac{2}{3} S_{x x}+\frac{1}{3} S_{y y}
$$

In Figures 13 and 14 we present some order parameters that can be calculated, namely, the deuterium order parameter and the bond order parameter, respectively. The chain order parameter was also calculated but has a behavior similar to the

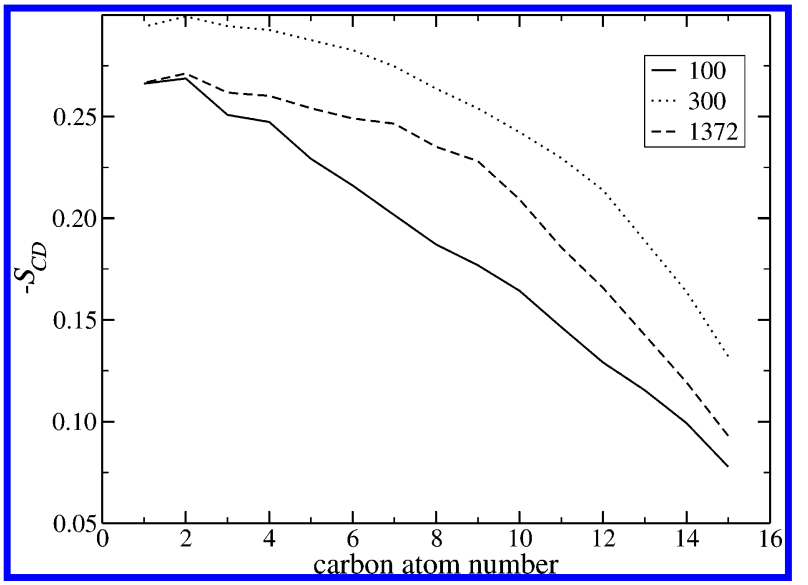

Figure 13. Average deuterium order parameter as a function of the carbon atom number. The water slab is located on the left-hand side and the oil slab on the right.

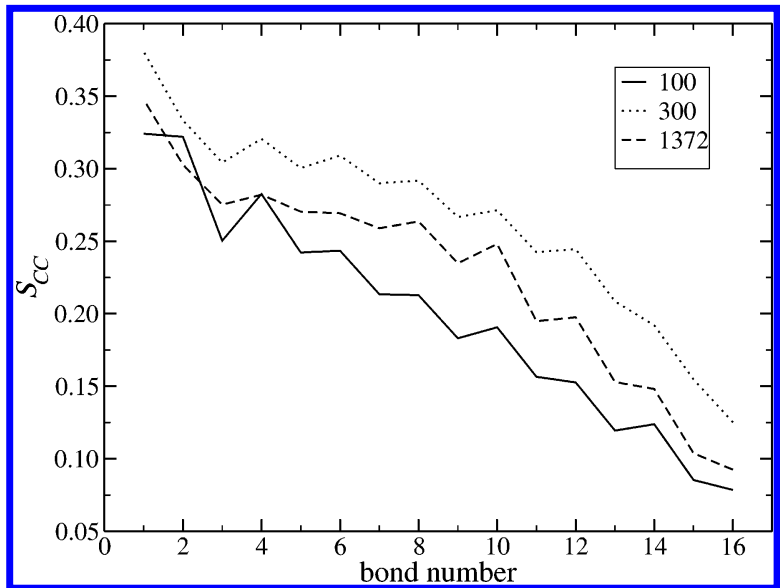

Figure 14. Average bond order parameter as a function of the carbon atom number. The water slab is located on the left-hand side and the oil slab on the right.

deuterium order parameter. For all the systems we find an increase in the randomness of the orientation of the molecular axes as one goes along the tails from the heads to the terminal methyl group. For all the systems, the chain order parameter and the deuterium order parameter suffer a slight increase from the first to the second molecular axis, near the CTA heads. The order parameters for the system containing 1372 DCE molecules have an intermediate value when compared with the other two systems. For this system, we can see that the order parameter curve is steeper (the absolute slope value increases) after the ninth molecular axis, and the order parameter changes more rapidly. For the system containing 300 DCE molecules, this change is not so clearly seen, since the curve has a round shape. Nevertheless, the difference between the first and last order parameter values is similar for all three systems. The bond order parameter curve has a general dependence on the bond number similar to the one described for the other order parameters on the carbon atom number. This order parameter also has a zigzag shape, typical of this property and similar to the one found for other systems. ${ }^{21}$ The values obtained for these order parameters are in good agreement with available data, namely, for lipid chains $^{33}$ or computer simulation data obtained for dodecyl sulfate $^{34}$ or phosphatidylcholine monolayers. ${ }^{17}$

The in-plane mean square displacement was calculated, and the results are presented for the CTA terminal methyl group in Figure 15 and for the nitrogen atom in Figure 16. The results for the Langmuir interface are also presented. 


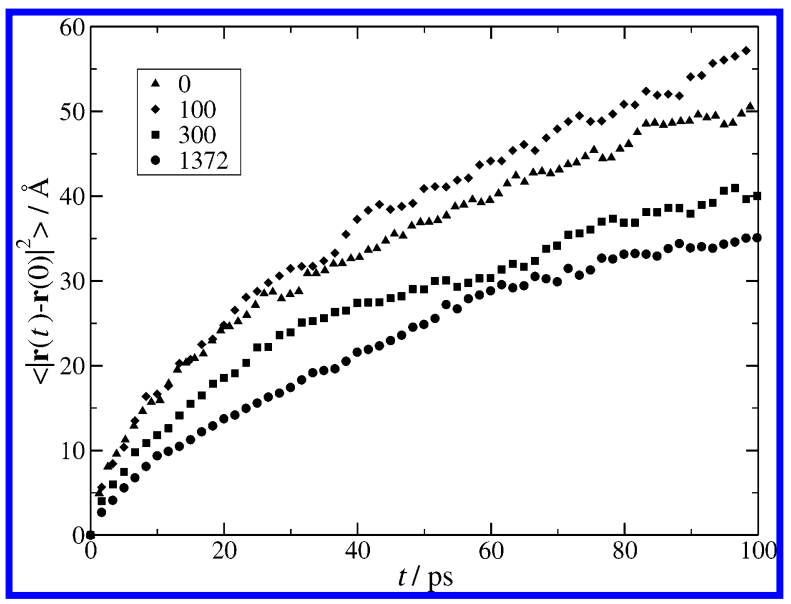

Figure 15. Mean square displacement of the CTA terminal methyl group for the Langmuir and water/DCE interfaces and for the systems containing 100 and 300 DCE molecules.

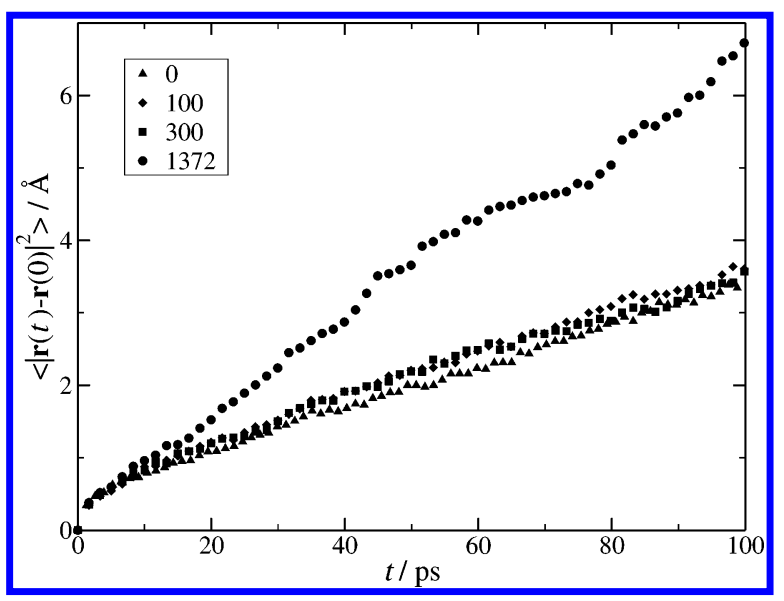

Figure 16. Mean square displacement of the CTA nitrogen atom for the Langmuir and water/DCE interfaces and for the systems containing 100 and 300 DCE molecules.

Using the last $50 \mathrm{ps}$ values of the averaged $100 \mathrm{ps}$ series to fit the results using the Einstein relation, ${ }^{21,35}$ we have obtained values of $(7.05 \pm 0.04) \times 10^{-6} \mathrm{~cm}^{2} \mathrm{~s}^{-1},(8.56 \pm 0.05) \times 10^{-6}$ $\mathrm{cm}^{2} \mathrm{~s}^{-1},(6.37 \pm 0.05) \times 10^{-6} \mathrm{~cm}^{2} \mathrm{~s}^{-1}$, and $(4.68 \pm 0.04) \times$ $10^{-6} \mathrm{~cm}^{2} \mathrm{~s}^{-1}$ for the in-plane diffusion coefficient of the CTA terminal methyl group at the Langmuir interface and for the systems containing 100, 300, and 1372 DCE molecules, respectively. As expected, the diffusion coefficient is on average inversely proportional to the number of DCE molecules present in the system (thickness of the film). Although the results could be grouped in two different groups, one with a relatively large quantity of DCE present and the other without or with few DCE molecules, the largest diffusion coefficient is found for the system containing 100 DCE molecules, which constitutes an unexpected result.

The in-plane diffusion coefficients for the nitrogen atom are $(0.748 \pm 0.002) \times 10^{-6} \mathrm{~cm}^{2} \mathrm{~s}^{-1},(0.713 \pm 0.003) \times 10^{-6} \mathrm{~cm}^{2}$ $\mathrm{s}^{-1},(0.637 \pm 0.003) \times 10^{-6} \mathrm{~cm}^{2} \mathrm{~s}^{-1}$, and $(1.36 \pm 0.01) \times$ $10^{-6} \mathrm{~cm}^{2} \mathrm{~s}^{-1}$ for the Langmuir interface and for the systems containing 100, 300, and 1372 DCE molecules, respectively. We can see that all but the value found for the system containing bulk DCE molecules are similar. For this system, the in-plane diffusion coefficient is much larger than for the others.

Of all the calculated properties, the tilt angle distribution and the in-plane diffusion coefficient are the most sensitive to the amount of DCE present in the system. For the nitrogen in-plane diffusion coefficient we can say that a different regime is reached when there is some bulk DCE in equilibrium with the monolayer. Although the CTA chains are flexible, a perturbation in one end is easily propagated to the other. It seams that when there is bulk DCE, this bulk layer decreases the mobility of the terminal methyl group (there is a large amount of DCE molecules that hindered the movement of the methyl group), but in opposition, since the methyl group is more fixed, it can serve as a fixed point for the CTA molecule to rotate, increasing the nitrogen diffusion coefficient.

\section{Summary and Conclusions}

In this study we have examined DCE films with 100, 300, and 1372 DCE molecules spread on a hexadecyltrimethylammonium (CTA) chloride monolayer adsorbed on water. The systems with 100 and 300 DCE molecules have a 1:1 and 1:3 CTA/DCE ratio, while the system containing 1372 DCE molecules can be regarded as a CTA monolayer adsorbed at the water/DCE interface. We have verified that the addition of a small amount of DCE molecules can notoriously change the $\sigma_{\mathrm{c}}$ (width of the chains distribution) and $\delta_{\mathrm{ch}}$ values (distance between the distribution centers of the CTA heads and tails). Nevertheless, the largest $\sigma_{\mathrm{c}}$ and $\delta_{\mathrm{ch}}$ values were obtained for the system containing 300 DCE molecules. This is unexpected, since the largest value was supposed to be obtained for the system containing 1372 DCE molecules, which corresponds to a macroscopic limit model of the water/DCE interface.

When comparing the widths of the water and CTA heads distributions, we have verified that the largest contact between the CTA heads and the water slab occurs at the Langmuir interface, and this contact decreases with the number of DCE molecules present in the system.

For the tilt angle distribution we have also found large differences. To change the mean value and the maximum probability angle, 100 DCE molecules are enough. On the other hand, we have found that more than 100 DCE molecules are needed to obtain a more similar distribution for CTA heads that penetrate more and less into the water slab. This means that the amount of DCE molecules must be enough to cover the CTA tails to obtain more similar distributions.

When analyzing the dihedral conformation state along the CTA chains, we have found an unexpected behavior for the system containing 300 DCE molecules due to the largest percentage of trans conformations found. The system containing 100 DCE molecules has the minor percentage, whereas the system containing 1372 DCE molecules has an intermediate position.

Analyzing the order parameters for some molecular axes along the CTA chains, we have verified that the system containing 100 DCE molecules presents the largest randomness in the orientation of the molecular axis just followed by the system containing 1372 DCE molecules.

The in-plane mean square displacement and the diffusion coefficient are very dependent on the number of DCE molecules present in the system. The in-plane mean square displacement for the nitrogen atom is very similar between the different systems studied, except for the system corresponding to the water/DCE interface. The mean square displacements are very similar between the systems containing 300, 100, or no DCE molecules. For the terminal methyl group of the CTA chain, the mean square displacement decreases with the addition of DCE molecules.

The unexpected behavior of the system with 300 DCE molecules poses an interesting problem that can suggest a nonideal behavior or, because of the small amount of DCE molecules, can point in the direction of carefully handled 
CTA Chloride Monolayers in Contact with Oil Films

polarization effects simulations. We expect to study this effect in more detail in a future work.

Acknowledgment. D.J.V.A.d.S. thanks the Programa PRAXIS XXI for a doctoral scholarship (BD/15576/98).

\section{References and Notes}

(1) Swalen, J. D.; Allara, D. L.; Andrade, J. D.; Chandross, E. A.; Garoff, S.; Israelachvili, J.; McCarthy, T. J.; Murray, R.; Pease, R. F.; Rabolt, J. F.; Wynne, K. J.; Yu, H. Langmuir 1987, 3, 932.

(2) Whitesides, G. M.; Laibinis, P. E. Langmuir 1990, 6, 87.

(3) Lu, J. R.; Thomas, R. K. J. Chem. Soc., Faraday Trans. 1998, 94 , 995.

(4) Miller, R.; Fainerman, V. B. In Handbook of Surfaces and Interfaces of Materials; Nalwa, H. S., Ed.; Academic Press: San Diego, CA, 2001; Vol. 1, p 383.

(5) Brevet, P. F.; Girault, H. In Liquid-Liquid Interfaces: Theory and Methods; Volkov, A. G., Deamer, D. W., Eds.; CRC Press: Boca Raton, FL, 1996; p 103.

(6) Messmer, M. C.; Conboy, J. C.; Richmond, G. L. J. Am. Chem. Soc. 1995, 117, 8039 .

(7) Lu, J. R.; Thomas, R. K.; Penfold, J. Adv. Colloid Interface Sci. 2000, 84,143

(8) Steel, W. H.; Damkaci, F.; Nolan, R.; Walker, R. A. J. Am. Chem. Soc. 2002, 124, 4824 . 4933.

(9) Beildeck, C. L.; Steel, W. H.; Walker, R. A. Langmuir 2003, 19 ,

(10) Zhang, X.; Cunningham, M. M.; Walker, R. A. J. Phys. Chem. B 2003, 107, 3183.

(11) Zhang, X.; Steel, W. H.; Walker, R. A. J. Phys. Chem. B 2003, $107,3829$.

(12) Linse, P. J. Chem. Phys. 1987, 86, 4177.

(13) van der Ploeg, P.; Berendsen, H. J. C. Mol. Phys. 1983, 49, 233.

(14) van Buuren, A. R.; Marrink, S.-J.; Berendsen, H. J. C. J. Phys. Chem. 1993, 97, 9206.

(15) Benjamin, I. Science 1993, 261, 1558.
(16) Benjamin, I. Chem. Rev. 1996, 96, 1449.

(17) Dominguez, H.; Smondyrev, A. M.; Berkowitz, M. L. J. Phys. Chem. B 1999, 103, 9582.

(18) Fernandes, P. A.; Cordeiro, M. N. D. S.; Gomes, J. A. N. F. J. Phys. Chem. B 1999, 103, 1176.

(19) Fernandes, P. A.; Cordeiro, M. N. D. S.; Gomes, J. A. N. F. J. Phys. Chem. B 2000, 104, 2278.

(20) dos Santos, D. J. V. A.; Gomes, J. A. N. F. ChemPhysChem 2002 $3,946$.

(21) dos Santos, D. J. V. A.; Gomes, J. A. N. F. Langmuir 2003, 19, 958.

(22) Conboy, J. C.; Messmer, M. C.; Richmond, G. L. J. Phys. Chem. 1996, 100,7617

(23) Conboy, J. C.; Messmer, M. C.; Richmond, G. L. J. Phys. Chem. B 1997, 101, 6724 .

(24) Miranda, P. B.; Shen, Y. R. J. Phys. Chem. B 1999, 103, 3292.

(25) Lu, J. R.; Thomas, R. K.; Binks, B. P.; Fletcher, P. D. I.; Penfold, J. J. Phys. Chem. 1995, 99, 4113.

(26) Lu, J. R.; Thomas, R. K.; Aveyard, R.; Binks, B. P.; Cooper, P.; Fletcher, P. D. I.; Sokolowski, A.; Penfold, J. J. Phys. Chem. 1992, 96 10971

(27) Lu, J. R.; Li, Z. X.; Thomas, R. K.; Binks, B. P.; Crichton, D.; Fletcher, P. D. I.; McNab, J. R.; Penfold, J. J. Phys. Chem. B 1998, 102, 5785 .

(28) Downer, A.; Eastoe, J.; Pitt, A. R.; Simister, E. A.; Penfold, J. Langmuir 1999, 15, 7591.

(29) Berendsen, H. J. C.; Postma, J. P. M.; van Gunsteren, W. F.; Hermans, J. In Intermolecular Forces; Pullman, B., Ed.; D. Reidel Dordrecht, The Netherlands, 1981; p 331.

(30) Jorgensen, W. L.; McDonald, N. A.; Selmi, M.; Rablen, P. R. J. Am. Chem. Soc. 1995, 117, 11809

(31) Smith, D. E.; Dang, L. X. J. Chem. Phys. 1994, 100, 3757.

(32) Egberts, E.; Berendsen, H. J. C. J. Chem. Phys. 1988, 89, 3718.

(33) Sheng, Q.; Shulten, K.; Pidgeon, C. J. Phys. Chem. 1995, 99, 11018.

(34) Dominguez, H.; Berkowitz, M. L. J. Phys. Chem. B 2000, 104, 5302.

(35) Allen, M. P.; Tildesley, D. J. Computer Simulation of Liquids; Oxford University Press: Oxford, UK, 1990. 